\title{
QUELLE GOUVERNANCE POUR LA CULTURE ET LE SECTEUR CULTUREL?
}

\author{
DOCUMENT DE TRAVAIL POUR LE CAMPUS \\ EURO-AMÉRICAIN DE \\ Las Palmas de Gran CANaria \\ (30 NOVEMBRE - 3 DÉCEMBRE 2010)
}

\section{Raymond Weber \\ UNESCO - Conseil de l'Europe - Collège d'Europe à Bruges}

RÉSUMÉ : Le thème de la gouvernance culturelle est abordé sous une triple approche : approche historique ; approche par la coopération au développement et les relations internationales ; approche par la société du savoir, l'économie créative et les TIC. Aujourd'hui, les politiques culturelles, en se fondant sur les droits culturels et sur une autre "gouvernance", pourront mieux répondre aux défis du monde de demain.

Mots-CLEFs : bonne gouvernance, gouvernance mondiale, coopération au développement, développement durable, société créative, Agenda 21 de la Culture.

RESUM: El tema de la governança cultural queda englobat en un enfocament triple: l'enfocament històric, l'enfocament de la cooperació al desenvolupament i les relacions internacionals, i l'enfocament de la societat del saber, l'economia creativa i les TIC. Avui en dia, les polítiques culturals, en basar-se en drets culturals $i$ en una altra «governança», podran respondre de manera més satisfactòria als desafiaments del demà.

Paraules Clau: bona governança, governança mundial, cooperació al desenvolupament, desenvolupament sostenible, societat creativa, Agenda 21 de la Cultura. 
RESUMEN: El tema de la gobernanza cultural queda englobado en un enfoque triple: el enfoque histórico, el enfoque de la cooperación al desarrollo y las relaciones internacionales, y el enfoque de la sociedad del saber, la economía creativa y las Tic. Hoy en día, las políticas culturales, al basarse en derechos culturales y en otra «gobernanza», podrán responder de manera más satisfactoria a los desafíos del mañana.

Palabras ClaVe: buena gobernanza, gobernanza mundial, cooperación al desarrollo, desarrollo sostenible, sociedad creativa, Agenda 21 de la Cultura.

ABSTRACT: The question of cultural governance is dealt with from three approaches: the historical approach, the development cooperation and international relations approach, and the knowledge society, creative economy and ICT approach. Today, cultural policies, based on cultural rights and on other "governance", will be better placed to meet the challenges of tomorrow.

KEYWORDS: good governance, world governance, development cooperation, sustainable development, creative society, Agenda 21 for Culture.

\section{Introduction}

Q

ue l'on parle de management d'entreprise, de coopération au développement, de relations internationales ou encore de politique culturelle, le terme « gouvernance » semble prendre, surtout depuis les années 1990, une importance grandissante tant dans le débat public que dans les stratégies et politiques adoptées au niveau local, national et international. Naviguant entre le monde des experts et celui des universitaires, entre vecteur d'analyses novatrices et simple déclinaison de l'idiome libéral, entre la conception d'un instrument technique de gestion et celle d'une vision citoyenne de la démocratie, ce « mot-valise », ce buzzword, est néanmoins devenu aujourd'hui un paradigme incontournable pour les politiques de développement économique, social et culturel, voire un vecteur de formulation d'un droit, national et international, nouveau. 
Le besoin d'une autre gouvernance est sans doute né d'un mode d'organisation du politique qu'on pourrait qualifier de stato-centré. Dès le milieu des années 1970, M. Crozier, S. Huntington et J. Watanuki évoquent une « crise des démocraties » occidentales qui s'exprime essentiellement par l'incapacité des États à faire face à l'ensemble des demandes sociales qui leur sont transmises. De cette crise des démocraties modernes, on passe, dans les années 1990, à une « crise de gouvernabilité », dans le cadre d'une double remise en cause, d'une part des conditions mêmes de production des politiques publiques et, d'autre part, de la légitimité de la puissance publique. Ce qui est critiqué alors, ${ }^{1}$ c'est le mode d'agrégation des préférences basé sur la légitimité légale-rationnelle monopolisée par l'État et ses administrations (Habermas, 1997), la confiance même dans le politique et dans sa capacité à traiter l'ensemble des problèmes des sociétés modernes et l'émergence d'une société civile de plus en plus revendicatrice en termes d'organisation du pouvoir (Keane, 1998), la fragmentation des systèmes décisionnels à la suite de la modification de la structure interne des États du fait des réformes décentralisatrices et des dynamiques fédéralistes (Loughlin, 2001), l'émergence de nouvelles problématiques (environnement, exclusion, intégration, sécurité, etc.) ne se prêtant plus à un traitement par des politiques sectorielles, mais nécessitant une intégration des approches et la recherche de synergies entre institutions et acteurs dont les logiques d'action, les cultures et les temporalités ne se recoupent pas (Duran et Koenig, 1996), la consolidation de nouveaux territoires d'action collective, à Porto Alegre ou dans les métropoles au sein desquels des mouvements sociaux avaient, dès les années 1970, fait le procès d'une intégration économique " par le haut " (Hamel, Lustiger-Thaler, Mayer, 2000) et, enfin, la redéfinition de la citoyenneté, dans sa traduction libérale et universaliste, par des groupes sociaux revendiquant un traitement communautaire permettant, selon eux, de passer outre à des politiques reposant sur la discrimination de groupes dominés (en fonction du genre, de la langue, des origines ethniques, des pratiques religieuses, de l'orientation sexuelle, etc. [Beiner et Norman, 2001]).

Pour Wikipédia, la gouvernance est « un mouvement de « décentrement » de la prise de décision, avec une multiplication des lieux et des acteurs im-

1. Cfr Jouve, B. (2007) : Les autres références se retrouvent dans la bibliographie à la fin de l'étude de M. Jouve. 
pliqués dans cette décision. Il renvoie à la mise en place de nouveaux modes de régulation plus souples, fondés sur le partenariat entre différents acteurs »».

Entre good governance, global governance, corporate governance et multilevel governance, on peut dégager, malgré un flou persistant, quelques caractéristiques :2

- La gouvernance se conçoit comme un mode de gestion d'affaires complexes, dans lequel les acteurs principaux se déploient sur le même plan, à l'horizontale sinon à égalité.

- La bonne gouvernance commande de gérer les affaires publiques comme si leur traitement ne devait pas différer sensiblement des affaires privées.

- Ce rôle de simple agent régulateur de l'ancienne puissance publique se trouve justifié par la croyance que les sociétés ou les relations entre pays se trouvent régies par des mécanismes d'auto-ajustement apparentés à ceux du marché sur le plan économique.

- On pourrait distinguer quatre niveaux de gouvernance :

- Le méta-niveau: vision politique et légitimation/mobilisation ; stratégies d'intégration multilatérales.

- Le macro: réforme des institutions politiques et changement démocratique des structures; institutions d'intégration régionale et droit.

- Le méso : régulation politique et capacité concurrentielle ; transfert de know how et capacity building ; interventions sectorielles.

- Le micro : organisation de systèmes régulateurs et modernisation administrative ; participation des citoyens et empowerment de la société civile.

- Se situant par conséquent dans une relation horizontale répudiant la vieille hiérarchie verticale qui garantissait le privilège de l'État souverain, les acteurs décisifs des dispositifs de gou-

2. Cfr Hermet, G. ; A. Kazancigil et J.-F.Prud'homme (2005). 
vernance se recrutent ou se choisissent entre eux, essentiellement par cooptation.

- La gouvernance correspond à un processus de décision toujours révocable et provisoire.

- Selon la logique néolibérale de la gouvernance, les décisions ne sont plus le produit d'un débat et d'une délibération, mais plutôt le résultat de négociations, voire de marchandages et de trocs entre les différentes parties.

- La logique de cooptation de la gouvernance s'applique particulièrement bien aux politiques sectorielles, dans une perspective néocorporatiste.

- La gouvernance est un mode de gestion qui tend à se codifier au regard de normes ou de « codes de conduite » négociés, plutôt que de lois votées en vertu du principe majoritaire ou issues d'une tradition jurisprudentielle illustrée par le Common Law.

La gouvernance, qu'est-elle finalement ? Un miroir aux alouettes, une simple technique de management mise en place par les institutions de Bretton-Woods pour pouvoir mieux gérer la mondialisation économique, selon une idéologie néo-libérale ou, au contraire, une utopie mobilisatrice qui nous permettrait de refonder une démocratie participative et inclusive, se fondant sur des réseaux de participation et de coopération citoyennes? Ou, peut-être, la gouvernance est-elle les deux à la fois ?

Cette étudevoudrait essayer de mieux situerles enjeux et de poserquelques questions auxquelles chacun doit répondre en fonction de son contexte socio-politico-culturel. Elle ne propose ni un kit de «prêt à penser », ni de " boîte à outils », avec des recettes toutes faites. L'analyse proposée est faite à partir d'une vue européenne et est marquée par l'expérience professionnelle de l'auteur qui, après avoir travaillé dans les champs de la culture, de la coopération au développement et du développement durable, essaie aujourd'hui de faire interagir dynamiquement ces trois «piliers » et de repenser la gouvernance comme une gouvernance démocratique, c'està-dire un moyen pour les sociétés d'élaborer leurs propres modalités de gouvernance. 
Après avoir précisé trois approches (approche historique, approche « développement » et approche « société du savoir »), le document développera la manière dont la gouvernance devient aujourd'hui un élément central de la politique culturelle.

\section{Une approche historique}

Si parler de «gouvernance culturelle » semble être un phénomène assez récent, la problématique plus générale de la gouvernance ou du « bon gouvernement » remonte loin dans l'histoire et retrouve même des racines africaines. Prenons trois exemples qui ont exercé - et qui continuent à le faire - une grande influence sur le vivre-ensemble des régions concernées :

\section{- La Charte de Kurukan Fuga (1236)}

La Charte de Kurukan Fuga (localité située dans le Cercle de Kangala, au sud de Bamako, capitale du Mali) est un ensemble de 44 « décisions » et « recommandations » prises par l'assemblée des 12 alliés du futur empereur mandingue Soundjata Keïta, au lendemain de la victoire, à Kirina, sur le roi sorcier Soumaoro Kanté. Cette sorte de Convention, sous forme de consignes consensuelles devant régir la vie publique sous l'Empire qui venait de naître, veut dépasser les années de guerres atroces et constituer une base nouvelle pour l'organisation de la société, l'exercice du pouvoir, la gestion des conflits et la mise en œuvre de la coexistence pacifique, en prônant les valeurs de la tolérance, de l'hospitalité, ainsi que des droits et devoirs de la personne humaine. La sanankunya (parenté à plaisanterie), ${ }^{3}$ le droit d'aînesse, la reconnaissance du rôle de la femme dans la société constituent les piliers de l'édifice social tel que prévu dans la Charte.

Citons quelques-unes de ces « décisions »:

- « chacun a le droit à la vi et à la preservation de son intégrité physique »;

3. La parenté à plaisanterie est une pratique sociale qui autorise les membres d'une même famille ou des membres de certaines ethnies entre elles, à se moquer ou à s'insulter, et cela sans conséquences : ces affrontements verbaux sont en réalité des moyens de décrispation sociale et de gestion de conflits. 
- «n'offensez jamais les femmes, nos mères »;

- «les femmes, en plus de leurs préoccupations quotidiennes, doivent être associés à tous nos gouvernements »;

- «ne faites jamais du tort aux étrangers »;

- «ne vous trahissez jamais entre vous. Respectez la parole d'honneur »;

- « venons en aide à ceux qui en ont besoin ${ }^{4}{ }^{4}$

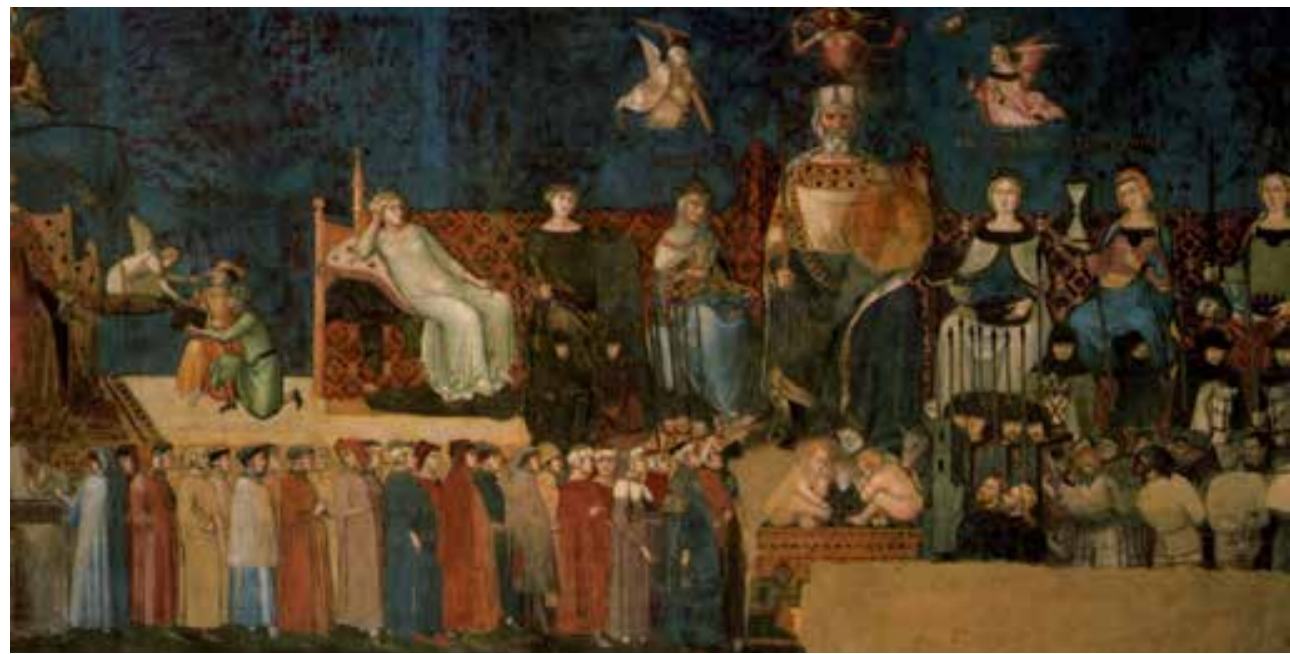

Illustration 1 : Allégorie du Bon Gouvernement, par Ambrogio Lorenzetti (Sienne, 1337-1339)

C'est de 1337 à 1339 que le grand peintre de l'École de Sienne, Ambrogio Lorenzetti, peint une série de fresques sur les murs de la Sala dei Nove (Salle des Neuf, c.-à-d. salle de réunion du gouvernement de Sienne), appelée aussi Sala della Pace (Salle de la Paix) du Palazzo Pubblico de Sienne.

La fresque principale, Allegoria del Buon Governo, se lit de gauche à droite et du haut vers le bas. La Sagesse ailée tient un livre fermé (le Livre

4. La Charte de Kurukan Fuga, qui se fonde sur la Charte du Mandé (1222), a été transmise de génération en génération par les djélis, dépositaires des traditions en pays malinké. Nous nous fondons ici sur une version collectée en Guinée à l'issue d'un atelier régional de concertation entre communicateurs traditionnels (essentiellement les griots) et modernes (essentiellement les animateurs des radios locales), avec l'aide de traditionnalistes et d'experts (Kankan, mars 1998). Rappelons, par ailleurs, que le site et la Charte de Kurukan Fuga ont été classés par l'unESCO, en mars 2009, comme étant de « Outstanding Universal Value », 
de la Sagesse) et la poignée d'une balance dont les plateaux sont suspendus à sa droite et à sa gauche, avec deux anges, entourant la Justice et représentant la justice distributive et la justice commutative. En-dessous se tient la Concorde, munie d'un rabot (pour aplanir les disputes et controverses) et passant deux cordes serrées ensemble à 24 bourgeois de la ville qui s'avancent con le corde (avec les cordes, dans la concorde) qui la transmettent à leur tour à cette imposante figure du Vieillard, vêtu de blanc et de noir, c.-à-d. des couleurs de la ville de Sienne et symbolisant tant la Commune que le Bien Commun. Au-dessus de sa tête, on retrouve les vertus théologales de Foi, de Charité et d'Espérance. Autour de lui, les « conseillères » Courage/Force, Prudence, Tempérance, Justice, Magnanimité et, surtout, cette figure de la Paix, tout en blanc, qui en impose par sa beauté et par cette " centralité » qui fait le lien entre les deux parties du tableau.

Sur les deux autres murs, on trouve les Effets du Bon Gouvernement dans la ville et à la campagne, ainsi que les Effets du Mauvais Gouvernement, dans la ville et à la campagne. Ces trois fresques constituent d'abord une composition, par les moyens propres de la peinture, d'une conception de l'espace public, du temps de l'histoire et des périls de la subversion de l'esprit public.

Mais elles traduisent aussi la promesse des Neuf de faire participer le peuple au gouvernement (les cordes, symboles de l'union entre la Sagesse, la Justice, la Concorde et le Bien commun, à travers les 24 bourgeois citoyens). En ce sens, elles « sécularisent» deux principes de morale politique, la justice et la subordination de l'intérêt privé au bien commun, avec, en arrière-plan, la genèse philosophique de l'idée moderne de démocratie, passant ainsi de la Civitas Dei de Saint Augustin à Saint Thomas et à la tradition aristotélicienne. $\mathrm{Au} 20^{\mathrm{e}}$ siècle, on retrouve, pour ainsi dire, cette préoccupation du bon et du mauvais gouvernement dans les fresques murales d'un Diego Rivera (Mexique, 1886-1957), notamment au Palais National de Mexico (1929-1935), à l'École d'Agriculture de Chapingo (1925-1927) et au Ministère de l'Éducation Publique (1923). 


\section{— Le Val d'Orcia et son modèle de « bonne gouvernance »}

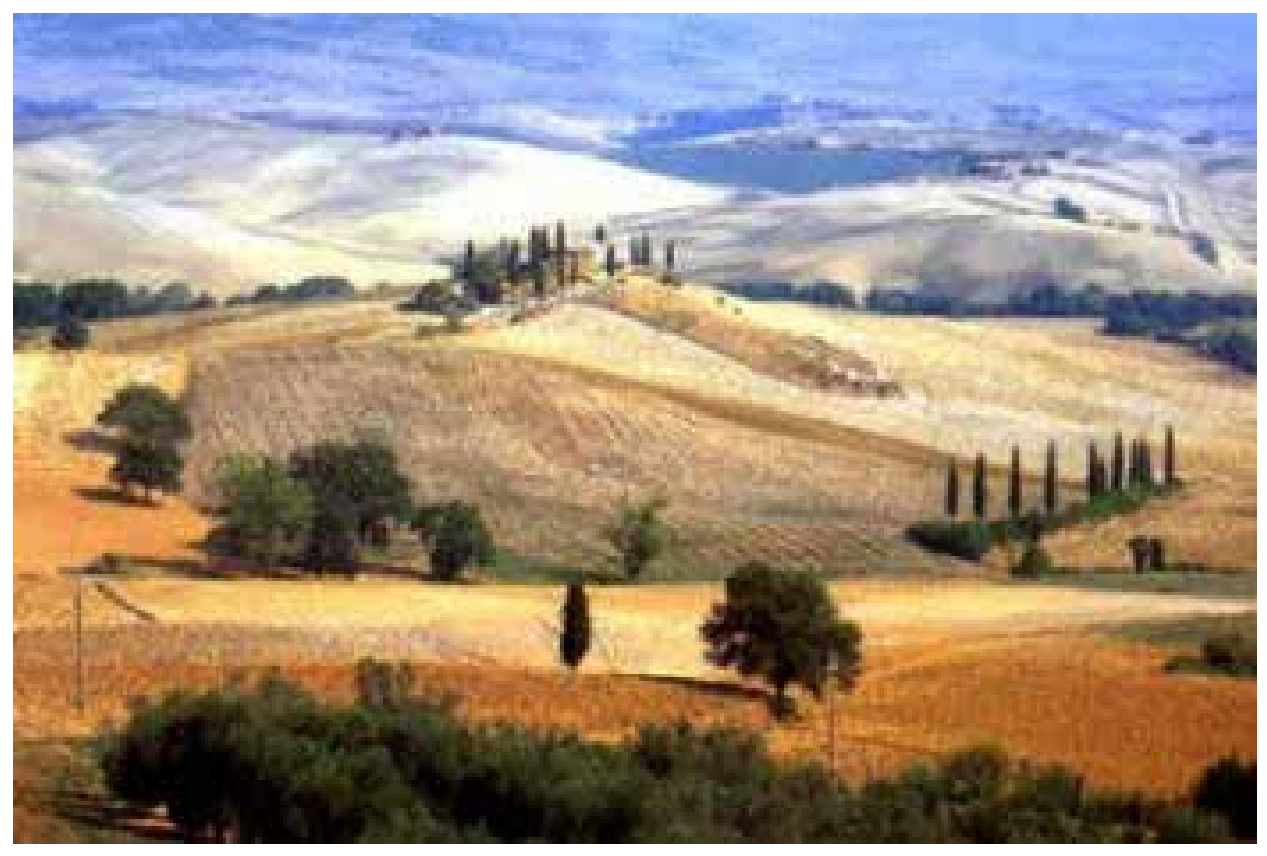

Illustration 2 : Val d'Orcia

Le paysage de la vallée de l'Orcia fait partie de l'arrière-pays de Sienne, en Italie. Il a été classé patrimoine mondial par l'UNESCO en 2004. Dans la justification d'inscription sur la Liste du Patrimoine Mondial, on peut lire ceci :

La Vallée de l'Orcia est un reflet exceptionnel de la réécriture du paisage durant la Renaissance qui illustre les idéaux de bonne gouvernance et la recherche esthétique qui a présidé à sa conception. Ce paysage a été célébré par des peintres de l'École de Sienne qui s'est épanouie à l'époque de la Renaissance. Des images de la vallée de l'Orcia, images d'un paysage idéal où l'homme vit en harmonie avec la nature, sont devenues des icônes de la Renaissance et ont eu une influence profonde sur le développement du paysagisme.

On pourrait évidemment ajouter d'autres exemples qui montreraient combien la gouvernance, comme approche globale, a déterminé l'organisation des sociétés et des paysages à travers les siècles, autour d'un certain nombre de valeurs : justice, tolérance, bien commun, espace public... 


\section{Une approche par la coopération au développement et les relations internationales : bonne gouvernance et gouvernance mondiale}

C'est à partir des années 1990 que la Banque Mondiale, pour tenir compte de la mondialisation qui se développe et de l'échec plus ou moins relatif des politiques d'aide au développement, parle de gouvernance et de « bonne gouvernance ». Alors que les recettes traditionnelles des institutions de Bretton-Woods tablaient sur les ajustements structurels, sur la privatisation et sur la dérégulation, la nouvelle orientation prise insiste sur l'importance d'un État efficient et de services publics efficaces, notamment en matière de santé et d'éducation. L'OCDE et son Comité d'Aide au Développement (CAD/DAC) y ajoutent, à partir de 1993, l'État de droit, la lutte contre la corruption, la réduction des dépenses militaires, le développement participatif, la démocratisation et les droits de l'homme. S'il y a apparemment une rupture avec le dogme néo-libéral de marché, la devise centrale reste celle d'une « efficiency through competition » de réformes gestionnaires et de l'établissement de conditions favorables pour l'économie privée, ainsi que la mise en place de politiques économiques et financières stables, orientées vers le développement et favorables au marché.

Si cet ensemble de principes de la bonne gouvernance est complété par la Déclaration de Paris (2005) et le Plan d'Action d'Accra (2007), textes qui vont renforcer les exigences en matière de résultats, d'efficience et d'efficacité, il n'en reste pas moins que quelques principes s'affirment qui vont au-delà d'un instrument technique au service d'un meilleur développement économique et qui vont marquer les relations internationales et d'autres domaines des politiques publiques : respect des droits de l'homme, renforcement de la société civile, mise en place de processus démocratiques, transparence des processus décisionnels, lutte contre la corruption, cohérence des politiques. S'y ajoutent aujourd'hui le développement durable dans ses dimensions de protection de l'environnement, d'efficacité économique et de justice sociale - , le empowerment des femmes, des minorités, des médias, etc., ainsi que la 
prise en compte des dimensions culturelles du développement. ${ }^{5}$ L'adoption, en 2006, au niveau de l'Union européenne, de la stratégie « la gouvernance dans le consensus européen. Vers une approche harmonisée au sein de l'UE » marque ici une étape importante, notamment dans le dépassement d'une approche purement gestionnaire : l'approche intégrée, la dimension politique et les différents niveaux de gouvernance (du local au global) doivent désormais être pris en compte dans les projets de gouvernance. Le dialogue, le pragmatisme en fonction du contexte et le renforcement des capacités locales se substituent à la promotion d'un modèle uniforme. Les dimensions normatives et prescriptives de la gouvernance sont rééquilibrées par une redécouverte de sa force analytique : elle s'affirme comme une approche permettant de mieux comprendre l'exercice du pouvoir légitime dans une société donnée et donc l'élaboration par les sociétés elles-mêmes de leurs propres modalités de gouvernance, en fonction des défis auxquels elles doivent faire face et dans le cadre des accords internationaux. Toutefois, malgré une vingtaine d'années d'utilisation de ce concept de bonne gouvernance, tant par les experts, les chercheurs, les membres de la société civile que par les politiques et les organisations bi et multilatérales, le champ de réflexion et d'action reste flou, alors même qu'une forte tendance novatrice se fait jour, questionnant non seulement la gouvernance des pays récipiendaires de l'aide, et celle des pays donateurs, mais aussi la gouvernance de l'aide elle-même. Bien plus, au-delà du développement, ce sont les défis communs de l'humanité qui se trouvent mis en perspective par la gouvernance : réalités locales $v s$ universalité, croissance économique $v s$ équité sociale, etc. Tout cela dans un espace de dialogue interculturel, porteur d'échanges, voire de convergences sur le rôle de l'État et celui des sociétés dans l'institutionnalisation du pouvoir.

Aujourd'hui, les idées-forces pour une reformation de la gouvernance semblent être les suivantes : ${ }^{6}$

5. Cfr la « Déclaration de Bruxelles » suite au colloque « culture et création, facteurs de développement » (avril 2009).

6. Cfr Bellina, S. ; H. Magro et de V. Villemeur (2008). 
- La nécessité de dépasser l'approche techniciste ainsi que les utilisations prescriptives et dogmatiques de la gouvernance et de son évaluation.

- Le prisme économique ne permet pas de prendre en compte tous les aspects et tous les enjeux de la gouvernance.

- Un consensus se dessine autour de la nécessité d'intégrer la dimension politique de la gouvernance aux analyses. La gouvernance apparaît ainsi comme une méthode d'analyse et de compréhension de l'exercice du pouvoir politique, ainsi que de l'action collective et de la gestion des conflits intrinsèques à toute société.

- Gouvernance et démocratie ne doivent pas être confondues. La gouvernance interroge la légitimité des pouvoirs au-delà des élections et de la notion de représentation.

- Le développement institutionnel de projets et politiques de développement au-delà de la technique, doit être le vecteur du pluralisme politique incarnant la pluralité sociale. Le renforcement des capacités, institutionnelles et humaines, est donc au cœur des politiques de gouvernance.

- La nécessité de dynamiques multi ou interacteurs, dans le cadre d'espaces de dialogue, fondées sur des réseaux ainsi que sur la capitalisation du savoir et des expériences, est fortement développée. De tels cadres favorisent l'élaboration de politiques publiques négociées, l'animation, du local au global, de l'institutionnalisation du pouvoir, autour de la définition de projets collectifs et de l'intérêt général.

- Les préoccupations relatives à la légitimité et à l'efficacité du pouvoir, dans le contexte de mondialisation et d'action publique participative, conduisent à mettre en avant l'approche territoriale de la gouvernance, le territoire étant l'espace d'articulation d'intérêts distincts et de rapports de force entre acteurs politiques, économiques et sociaux relevant de multiples échelles.

- La gouvernance locale, ancrée dans le territoire local, constitue l'espace privilégié de mobilisation des acteurs de la gouvernance : elle représente un échelon de vie, d'apprentissage de la citoyenneté, de 
confrontation des différents intérêts et des différentes sources de légitimité, de nombreuses dynamiques économiques (notamment informelles), de cohabitation interethnique et interreligieuse, etc.

- C'est dans l'articulation entre les différents échelons de gouvernance que chacun d'entre eux se renforce. La double dynamique d'affirmation de la gouvernance locale et de l'intégration régionale d'une part, de la gouvernance nationale et de la gouvernance globale d'autre part, est au cœur des processus de refondation politique de l'État et du « système international » : la définition du bien commun et la dynamique des biens publics mondiaux confirment que les défis posés par la gouvernance sont communs (environnement, eau, pandémies, démocratie, sécurité, diversité culturelle, etc.) et appellent à une gouvernance mondiale adaptée.

Reste une question pour laquelle les réponses sont loin d'être claires : quels critères pour évaluer la gouvernance ? Ceux qui ont accompagné les programmes d'ajustement et les programmes de réformes économiques ? On parle dès lors de bonne gouvernance quand le gouvernement est capable de faire passer les réformes. Sont pris en considération aussi le niveau d'ouverture commerciale d'un pays, ou la privatisation du système financier ou encore la réduction des services ou de l'emploi public.

Une conception plus néo-institutionnelle ? On pense alors aux libertés démocratiques, une presse libre, une justice indépendante, à l'intégrité et à la transparence. On essaye dès lors d'établir des critères permettant d'évaluer dans quelle mesure le système démocratique fonctionne comme une médiation efficace pour prendre des décisions et les mettre en œuvre dans des conditions d'efficacité, d'intégrité et de transparence.

Enfin, une conception pour laquelle l'important est de savoir comment une société construit ses propres capacités pour intervenir dans les problèmes essentiels qu'elle arrive à identifier ? Ce qui intéresse dès lors, ce sont les résultats dans trois domaines particuliers : l'intégration sociale, comme réduction de la pauvreté ; l'intégration politique, comme pluralisme et capacité d'intervention des citoyens sur les affaires publiques et, enfin, l'intégration culturelle, avec la capacité de gérer les conflits. 
Il semble être aujourd'hui entendu, tant dans les politiques de développement que dans les politiques culturelles, que :

- Les politiques de développement doivent assurer la gouvernance du secteur culturel proprement dit, en renforçant les institutions publiques et en encourageant la mise en œuvre de politiques culturelles prenant en compte la diversité culturelle et favorisant le développement culturel.

- Dans un souci de meilleure gouvernance, les programmes de renforcement institutionnel doivent insister sur la création de capacités institutionnelles. Au-delà de la dotation d'infrastructures et de renforcement budgétaire, il s'agit ici d'investir dans le capital humain, principale ressource non seulement du secteur culturel, mais aussi de la société du savoir.

- La gouvernance concerne aussi la gestion de la diversité culturelle et la construction de la citoyenneté. Tout comme les identités, définitivement multiples, la citoyenneté doit être considérée comme un espace ouvert de négociation permanente.

- La gouvernance doit encourager la participation des acteurs, de tous les acteurs - publics, privés et civils -, afin de garantir la pluralité et la diversité comme éléments de base de la vie démocratique ; la récupération de l'espace public par les citoyens joue un rôle décisif dans la création de sociétés participatives et inclusives. L'accès des citoyens à la culture est aujourd'hui un droit ${ }^{7}$ et une responsabilité des institutions publiques et de la société civile, susceptibles de donner naissance à des espaces de vivre ensemble favorisant la gouvernance et la cohésion sociale. ${ }^{8}$

- Le secteur culturel a un important rôle à jouer dans la promotion du développement social et culturel durable. « Fair Culture means realising cultural rights and including everyone in cultural signification, irrespective of age, gender, disability, or ethnic, religious and cultural

7. Cfr Article 27 de la Déclaration Universelle des Droits de l'Homme et la Déclaration sur les droits culturels.

8. Cfr Martinell, A. (2010). 
background. These are aspects that should also be guidelines for development cooperation $\gg .^{9}$

Toutes ces réflexions nous montrent que les chefs d'État et de gouvernement, en proclamant officiellement, en 2000, les Objectifs du Millénaire pour le Développement, ont eu tort de ne pas intégrer parmi les sept objectifs, ni dans le $8^{\mathrm{e}}$, qui concerne la coopération internationale, la culture et la gouvernance culturelle. Elles sont manifestement le lien qui manque pour assurer la cohérence et l'interaction entre les différents objectifs et pour créer une dynamique socio- politique de développement humain.

\section{Une approche par les droits culturels}

C'est autour du Groupe de Fribourg et de la Déclaration des droits culturels ${ }^{10}$ proclamée en mai 2007 à Fribourg et à Genève, que s'est développée toute une réflexion sur la gouvernance culturelle et la culture démocratique. La notion de « gouvernance culturelle » y apparaît selon l'orientation :11

- À la différence du gouvernement qui s'adresse à des individus et à des institutions, la gouvernance est un système de régulations qui vise des interactions. Le rapport gouvernants-gouvernés est remplacé par l'interaction d'acteurs individuels et institutionnels qui ont en partage la responsabilité du bien commun, et dont le jeu démocratique est garanti par les autorités publiques, sous le contrôle de tous les acteurs. Relevant, certes, du pragmatisme inhérent à toute gestion, une gouvernance est démocratique dans la mesure où elle assure l'interaction des acteurs sous le contrôle d'espaces publics.

- Cette rénovation de la culture démocratique, ou mise en scène de tous les acteurs, se fait dans un espace à plusieurs échelles. La mondialisation ne signifie pas que tout est global, mais qu'aucune activité politique ne peut être contenue dans un seul cadre politique : la

9. Cfr Fair Culture (2006).

10. $\mathrm{Cfr}<\underline{\text { www.unifr.ch/iiedh }}$ et www.droitsculturels.org $>$.

11. Cfr Meyer-Bisch P. et M. Bidault (2010). 
gouvernance pose constamment la question des échelles spatiales et temporelles (limites de la communauté politique concernée et durée envisagée du développement). La mondialisation n'est pas la réduction des échelles du politique à une seule, englobante : elle est leur interdépendance. Si les démocraties libérales sont fondées sur les droits civils et politiques, et si les démocraties sociales sont une interprétation des droits sociaux, Alain Touraine annonce un $3^{\mathrm{e}}$ étage qu'il décrit comme « un nouveau paradigme ». ${ }^{12}$ Si chaque droit de l'homme est, selon la formule d'Amartya Sen, « fin et moyen du développement », cela signifie que chaque groupe de droits est facteur de démocratisation : à la fois réalisation de la fin - la dignité des personnes - et moyen de réalisation - le développement des capacités. Le respect des droits culturels permet le développement des capacités individuelles primordiales, car ils sont la condition nécessaire pour que chacun puisse se relier aux œuvres et à autrui, qu'il soit nœud ou tisserand du tissu social. Ils sont aussi la condition pour que les acteurs sociaux alimentent par leur diversité de savoirs un espace public riche et diversifié.

La démocratie est-elle un cadre juridique qui permet de garantir le jeu des libertés civiles et du marché, ou est-elle un lien politique qui tend à développer l'ensemble des libertés? Une conception faible de la démocratie se limite aux processus électoraux, à la décision majoritaire et au règne du compromis. Dans une conception forte, le politique n'est plus un cadre pour les institutions, mais un lien qui relie les citoyens de façon interactive par l'intermédiaire des institutions sous leur contrôle permanent. Il ne s'agit pas seulement de lien social (pour favoriser les rencontres entre les groupes sociaux et culturels), mais de lien politique (favorisant le débat entre ses acteurs sur son fonctionnement et sur l'interprétation des valeurs fondatrices). La loi du compromis est remplacée par la recherche jamais achevée du consensus : c'est le débat permanent qui fait la légitimité démocratique, dans une « démocratie participative ».

À partir de cela, on peut tirer quelques « conclusions » :

12. Cfr Touraine, A. (2005). 
- L'exigence du respect des droits de l'homme (y compris les droits culturels) et des principes de l'État de droit nécessite l'invention de nouvelles régulations, non plus centrées, mais systémiques. Nous devons passer d'une pratique des réseaux à une régulation des systèmes. Les systèmes sociaux ne sont pas à prendre ici au sens de Niklas Luhmann (le « mutisme mutuel des systèmes »), mais comme des systèmes d'interrelations, au service des sujets, régulièrement contrôlés par eux, y compris dans leurs interdépendances. Dans cette perspective, la gouvernance culturelle n'est rien d'autre qu'une politique culturelle, comprise comme une participation de tous les acteurs culturels, non seulement à sa mise en œuvre, mais aussi à la définition de ses objectifs et de ses échelles.

- En tant que domaine du politique, le système culturel participe par ses interactions à la « production » d'une société, c'est-à-dire, à la constitution de ses acteurs et de leurs réseaux. Il est principe du politique puisqu'il est communication des savoirs et donc des identités. À la pluralité des formes de savoirs (sciences, valeurs, modes de vie, de production, de croyance, etc.) correspondent des systèmes sociaux, plus ou moins développés, plus ou moins autonomes et/ou interdépendants. La richesse de chaque système et celle de leurs connexions constituent le capital social et culturel d'une société que les politiques culturelles ont à gérer : à respecter et à faire fructifier au service du plus grand nombre.

- Établir les liens au savoir, notamment par les droits culturels, c'est remettre l'autonomie du sujet au centre, ainsi que celle des acteurs sociaux. Voilà l'enjeu éthique principal. Mais il y a aussi un enjeu méthodologique : une gouvernance culturelle est à la fois une politique sectorielle et une culture de l'ensemble du champ politique, dans une approche d'inclusion mutuelle.

- Une gouvernance démocratique suppose une définition toujours en débat, dans des espaces publics appropriés, des domaines et des acteurs : on peut parler ici de « triangulation des acteurs $»::^{13}$ acteurs pu-

13. Cfr Meyer-Bisch, P. (2010). 
blics (État et ses institutions, autres pouvoirs publics), acteurs privés (entreprises et secteur privé), acteurs civils (associations, organisations à but non lucratif, fondations). S'y ajoute la nécessité d'identification des circuits et de la cohérence des fonctions de chaque acteur, afin de pouvoir préciser les responsabilités et les responsabilisations (empowerment).

- La gouvernance culturelle signifie donc une cohérence entre les acteurs et les fonctions en vue d'objectifs communs (et choisis en commun). Les modes d'interaction, les processus de choix des objectifs, des acteurs et des fonctions, ainsi que les processus de contrôle définissent une gouvernance politique. Deux conditions à cela : que la gouvernance provienne de tous les individus et de tous les acteurs constitués. Et que cette gouvernance démocratique s'exerce en prenant l'accomplissement des droits humains comme moyens et comme fins.

\section{Une approche par la société du savoir, l'économie créative et les TIC (Technologies de l'Information et de la Communication)}

Société de l'information, société de la connaissance, société post-industrielle et post-moderne, société d'abondance et société de consommation, société en réseaux, capitalisme informationnel, capitalisme numérique : les concepts ne manquent pas pour traduire une évolution de nos sociétés, dans les cinquante dernières années, dont nous ne maîtrisons guère les aboutissants, mais dont un certain nombre de caractéristiques s'affirment un peu partout, au moins dans les pays "développés » et en émergence :

- La croissance du secteur des services et de l'économie créative.

- La centralité de l'information et de la connaissance comme facteurs de production.

- L'augmentation de la productivité grâce à la créativité et à l'innovation. 
- La naissance de nouveaux modèles de production économique, de régulation sociale et de vie culturelle.

- Le développement $d^{\prime}$ « intelligences collectives ».

- La métamorphose des organisations à l'ère numérique (Web 2.0.).

Concernant la gouvernance et la gouvernance culturelle, il y a nombre de conséquences plus ou moins immédiates, mais aussi des questions qui se posent et où on peine encore quelque peu à trouver les réponses adéquates :

- La généralisation des caractéristiques culturelles à une bonne partie de la production économique, telle qu'elle découle de l'économie créative, ne va-t-elle pas entraîner une dilution, concomitante à cette extension, de la spécificité et de la « densité » du champ des arts et de la culture ? Une problématique « culturalisation » de l'économie ne risque-t-elle pas d'entraîner aussi une plus grande intégration des industries culturelles, voire du secteur culturel tout entier, dans une logique capitaliste?

- La dématérialisation de la production et la mise en réseau des acteurs ouvrent des perspectives dont on ne fait encore que commencer l'exploration : libération de la parole et possibilité pour toute personne connectée d'exercer de façon significative son droit à la liberté d'expression ; essor sans précédent du travail coopératif, notamment pour les artistes, les chercheurs scientifiques et les acteurs culturels ; possibilité, par la fluidité du numérique, de faire circuler avec une facilité inédite des textes, des sons, des images fixes ou animées, offrant ainsi des occasions quasi illimitées de diffusion, mais aussi de reprise et de réinterprétation.

- Le savoir et l'intelligence partout distribués, sans cesse valorisés, coordonnés en temps réel, aboutissent à une mobilisation effective des compétences et provoquent ainsi une révolution de communication entre les êtres humains. Internet nous oblige à expérimenter de nouvelles manières d'être ensemble, sur la base d'une éthique de l'intelligence collective qui consiste à entrelacer les points de vue 
différents ${ }^{14}$ et à développer apprentissage collectif, mémoire collective, compétence collective et responsabilité collective, dans un « système apprenant intelligent ».

- Tout cela bouleverse les métiers et les positions relatives des acteurs culturels, en faisant interagir différemment les différentes « postures » de l'individu constamment connecté : consommateur, artiste, acteur, citoyen...

- Il en résulte aussi une remise en cause assez radicale des filières de production et des procès de création, de production et de distribution, dans une métamorphose des organisations et dans une nécessité de les redéfinir par rapport à des « territoires communicants» et des « réseaux dits intelligents ».

- Il convient cependant d'insister sur les risques de fragilisation de ceux qui n'intégreraient pas les apports majeurs des technologies numériques et qui ne pourraient donc pas profiter des opportunités d'innovation et de croissance de l'économie créative.

- Tout en marquant des réticences vis-à-vis des théories d'un Richard Florida sur la " classe créative », il convient de souligner que ces nouveaux « créatifs culturels » présentent un certain nombre de caractérristiques qui peuvent devenir innovants aussi pour d'autres secteurs telles que : la logique de fonctionnement en réseau et la mise en place de formes de socialisation qui réunissent des ressources collectives tout en préservant l'autonomie et l'individuaité de chaque invididu. La connectivité et la force " compétitive $»^{15}$ des nouveaux métiers culturels ne doivent cependant pas nous faire oublier qu'ils restent souvent très précaires.

Les objectifs d'une politique culturelle pour une société créative (qui ne se contenterait pas de se centrer sur les forces du marché, mais qui viserait aussi les investissements en matière de démocratie sociale et culturelle) pourraient dès lors être :

14. Cfr Lévy, P. (2002).

15. Différence entre coopération et competition. 
- De développer les aptitudes à penser en dehors des sentiers battus (out the box thinking), ce qui suppose des possibilités d'échange et de débats pour définir des projets possibles, et les conditions pour les mettre en œuvre.

- D'élever le niveau des compétences et des capacités, en permettant aux employeurs, employés, élus, leaders civiques, de renforcer de manière continue leurs aptitudes.

- D'attirer tous ceux qui peuvent contribuer au développement, ce qui implique un environnement attractif (sans pour autant favoriser le brain drain dans les pays en développement).

- De favoriser le cadre spatial des villes, des métropoles et des régions. La gouvernance de la politique culturelle change ici de niveau et d'enjeu : il ne s'agit plus de voir comment un État central veille à ce que ses décisions se concrétisent au mieux sur le terrain, mais de faire en sorte que les acteurs d'un territoire conçoivent leur développement dans le cadre de contraintes globales et de péréquations possibles de ressources. On substitue dès lors à une politique culturelle institutionnalisée de haut en bas un véritable écosystème culture ${ }^{16}$ où les demandes, positions et opportunités des différents acteurs s'ajustent en permanence les unes aux autres, le rôle des pouvoirs publics étant de garantir les conditions qui rendent l'écosystème producteur de la valeur recherchée (création de sens et créativité), soutenable et résilient.

Mais tous les « effets » de la société du savoir et de la société créative sont démultipliés par la «révolution» des technologies de l'information et de la communication. Internet est considéré, à juste titre, comme une véritable révolution économique, politique et culturelle et provoque « un séisme dans la culture $»,{ }^{17}$ par l'opportunité offerte par de nouvelles pratiques qui changent notre rapport à l'information et à la connaissance, qui renouvellent les circuits de la diffusion culturelle et qui bousculent le statut de la création.

\footnotetext{
16. Cfr Greffe, X. (2010).

17. Cfr Le Glatin, M. (2007).
} 
Sur le plan économique, un modèle économique fondé sur la rareté matérielle est remplacé par un modèle économique fondé sur l'abondance immatérielle. La notion de bien commun artistique nous montre que nous ne sommes pas en présence d'une simple évolution technologique, mais bien d'une mutation de civilisation, d'une révolution d'ordre anthropologique, comportant une nouvelle manière de vivre ensemble, de communiquer, des rapports sociaux transformés, une représentation du monde et de la culture profondément transformée, des relations radicalement différentes avec l'espace et le temps. Plus encore que sur le plan économique, c'est sur ce plan sociétal qu'Internet apportera une extraordinaire libération d'énergie sociale et permettra une mutualisation des savoirs, le mixage et la création d'œuvres « collaboratives », ainsi que l'arrivée d'œuvres nomades. Il est bien possible que nous allions vers une culture qui ne se réduirait pas à un ensemble d'œuvres, mais se définirait principalement par des mécanismes de relations sociales régissant les contributions de plusieurs personnes à des œuvres communes et toujours inachevées. Sans que la singularité de son expression artistique ne soit niée, chaque individu est mobilisé dans une dynamique polyphonique. La dimension collective de la création offre en retour l'occasion d'en découvrir d'autres en soi par frottement à la subjectivité des différents participants.

La discussion sur Internet et les TIC nous montre que dans une société du savoir et dans une économie de l'immatériel, le rôle de ce cyberespace comme espace d'expression artistique et démocratique sera de plus en plus important et que nous sommes dans une mutation dont nous ne voyons pas encore tous les aboutissements. Il est donc urgent et important que la discussion afférente soit menée non seulement d'un point de vue technique ou économique, mais aussi du point de vue culturel et sociétal. Il semble indispensable, par ailleurs, que ce débat soit public, tant au niveau national qu'au niveau international (où il faut faire évoluer le droit international qui paraît largement obsolète en face des évolutions techniques).

\section{La gouvernance, enjeu des politiques culturelles}

Les politiques culturelles apparaissent, un peu partout dans le monde, en crise : crise de contenus (et d'objectifs), crise structurelle, crise de gouver- 
nance (et de processus de mise en œuvre). Alors qu'on attend beaucoup (trop ?) de la politique culturelle, notamment pour les impulsions qu'elle devrait donner à d'autres politiques (telles que la coopération au développement et les relations internationales, le développement économique, la justice sociale, l'éducation, l'aménagement du territoire, etc.), elle semble faire face à un triple dilemme : ${ }^{18}$

- Dilemme de sens et de signification, pour dégager des majorités politiques, voire des consensus, autour d'un certain nombre de finalités.

- Dilemme de compétence : quels sont aujourd'hui les rôles de l'État vis-à-vis des autres pouvoirs publics (régions, villes et communes), vers le secteur privé et vers le secteur civil (associatif), mais aussi dans un monde de plus en plus globalisé et médiatisé (organisations régionales et mondiales)?

- Dilemme de mise en œuvre, notamment en définissant les cadres juridiques, en dégageant des ressources et en aidant à donner forme aux relations et coopérations entre les différents acteurs de la vie culturelle.

Aujourd'hui, la culture agit de manière transversale dans la société, en irriguant des aptitudes à la créativité dans tous les secteurs. Par les nouvelles valeurs et références qu'elle produit, la culture contribue à des innovations sociales autant qu'économiques, et ce quel que soit le secteur concerné. La politique culturelle est transversale et doit prendre en considération l'ensemble des activités de la société, ainsi que les réseaux qui les développent. Ce qui reste cependant commun, c'est la production et la mobilisation des talents et compétences artistiques. ${ }^{19}$

Dans « une culture sans centre » et devant la multiplication des acteurs, ne faudrait-il pas dégager un nouveau « contrat culturel» entre les trois secteurs (public, privé et associatif), fondé sur la recherche de consensus autour d'un certain nombre de valeurs et de finalités culturelles, de la reconnaissance d'un pluralisme structurel et du principe de subsidiarité, ainsi que sur la

18. Cfr Scheytt, O. (2008).

19. Cfr Greffe, X. et S. Pflieger (2009). 
nécessité de dégager tant des ressources suffisantes que des espaces de liberté pour les expressions artistiques et culturelles ? Comment privilégier les approches bottom up et le fonctionnement et la coopération en réseau $?^{20}$

Dans un tel contexte, l'État et les pouvoirs publics tenteraient plus d'agir sur le contexte que de réguler, plus de convaincre que d'imposer, plus d'aider à faire (par l'éducation et par l'empowerment, notamment) que de faire euxmêmes.

Le Conseil de l'Europe a beaucoup travaillé sur les politiques culturelles et vient d'organiser, à Bruxelles (septembre 2010), une conférence sur le thème de « la culture et les politiques du changement $»{ }^{21}$

L'intention affichée est de lancer un processus de réexamen des approches actuelles de la politique et des pratiques culturelles en tenant compte des grands bouleversements mondiaux - y compris l'effet des restructurations économiques et la réduction des budgets publics consacrés à la culture, mais aussi d'autres évolutions qui se produisent sur le plan de la démographie, des questions liées à la diversité, de l'incidence des nouvelles technologies, des préoccupations environnementales et de la sécurité. Cette évolution suscite un large débat dans les pays européens sur le rôle des pouvoirs publics et du secteur privé dans la culture, mais aussi sur le mode de fonctionnement des institutions culturelles, le type et l'échelle des soutiens dont les artistes ont besoin, les nouvelles formes de partenariats et les approches plurielles pour relever les défis auxquels la politique culturelle au sens large est confrontée en Europe, au niveau local, régional, et paneuropéen.

On a sans doute parlé jusqu'ici trop peu des implications pratiques des grandes mutations économiques et sociétales au niveau local et national pour les responsables politiques et les praticiens en matière de politique culturelle. Les problèmes financiers sont manifestes dans un certain nombre de pays où il est prévu de réduire considérablement les budgets et les subventions des

20. Cfr La théorie de l'acteur-réseau, Latour, B. (2007).

21. Dans le cadre de son programme CultureWatchEurope et ensemble avec Culture Action Europe, le Conseil Economique et Social Européen, IFCCD (International Federation of Coalitions for Cultural Diversity), CultureLab, EUNIC (réseau des instituts culturels européens), Observatoire de Budapest, etc. 
institutions culturelles. L'opinion et les valeurs publiques sont aussi influencées par les problèmes environnementaux et d'autres priorités. Il s'agit donc de mettre l'accent, dans une perspective élargie, sur une nouvelle approche de la culture et de la politique culturelle qui pourrait être intégrée dans beaucoup de secteurs et d'intérêts différents. Par ailleurs, il semble hautement souhaitable de proposer des mesures pratiques clés et des propositions qui pourraient utilement être mises en œuvre à l'avenir par les gouvernements, les responsables politiques et les organisations de la société civile.

Dans ce contexte, il conviendrait sans doute aussi de parler des relations entre culture et développement durable. Le développement durable doit être considéré ici comme un nouveau projet de société, la trame d'une nouvelle étape pour l'organisation des activités humaines, une « règle du jeu » qui mettra (enfin) l'économique au service du développement social et de la lutte contre les inégalités, tout en économisant au maximum les ressources naturelles de la biosphère que nous savons limitées.

Ce projet, à contre-courant du libéralisme et du consumérisme dominants, est porteur d'une nouvelle culture qui doit irriguer les modes de vie, les modes de production et de consommation, la vie politique mais aussi les sciences, la pratique des arts, la littérature, la philosophie...

Dans cette perspective, le développement durable suppose une très large adhésion sociale qui nécessite en amont information, formation, réflexions et débats, échanges d'expériences. De même, la préservation de la diversité culturelle, la revalorisation des pratiques artistiques et culturelles et, de façon plus générale, toutes les richesses immatérielles que nous considérons essentielles à la construction de l'humain - en tant qu'outils de relation et de connaissance - sont au cœur de la problématique de développement durable.

En associant autour d'un enjeu commun « culture » et « développement durable », on pourra sans doute contribuer à tisser la trame d'une nouvelle étape pour l'organisation des activités humaines.

Toutes ces réflexions et ces travaux nous font insister sur les principes suivants d'une politique culturelle :

- La culture devra assumer son rôle central dans les politiques et stratégies de développement de la société. 
- La politique culturelle doit se fonder sur les valeurs fondamentales des droits de l'homme et des droits culturels.

- Les besoins culturels de la population, l'accès à la culture et la participation culturelle doivent constituer la base de toute politique culturelle.

- La politique culturelle vaut autant par ses processus que par ses contenus : elle doit réunir, en toute transparence, les différents acteurs autour d'objectifs centraux.

- Elle doit être prospective, en considérant les différents défis auxquels elle doit faire face comme autant de possibilités de se transformer et de se renouveler.

- Mobilité et intégration doivent être deux principes de base pour les artistes, les acteurs culturels et les instances politiques.

- Toute politique culturelle, pour atteindre la qualité et l'excellence, doit veiller à une professionnalisation accrue, à une formation de qualité et à un statut de l'artiste pleinement reconnu par la société. $^{22}$

Dans ce contexte assez nouveau, on pourrait définir la gouvernance culturelle comme suit : l'ensemble des voies et moyens par lesquels les citoyens et les institutions culturelles - publiques et privées - déterminent et régulent, dans des processus continus, leurs intérêts différents et initient des « agir » coopératifs. Le rôle de l'État, qui doit être « activant », ${ }^{23}$ y est primordial :

- Comme arbitre entre les différentes cultures, religions et intérêts divers dans une société déterminée.

- Comme médiateur, entre les créateurs et les publics, entre les artistes et les institutions, entre le secteur culturel et les autres secteurs, entre les secteurs public, privé et associatif.

22. Cfr Kathrin Merkle (2010).

23. En allemand : ein « aktivierender Staat»; cfr la Commission d'Enquête du Parlement allemand et Scheytt, O. (2008). 
- Comme facilitateur, il doit veiller au développement d'une société créative, par l'empowerment des différents acteurs (et notamment des plus faibles), par la mise en place de systèmes d'enseignement et de formation de qualité. ${ }^{24}$

Comme il n'y a pas - heureusement - de modèle unique de gouvernance culturelle, les formes varient : forums, plate-formes, conseils, « parlements », " clusters », etc. Ce qui est commun, c'est la libre discussion dans un espace public, la participation citoyenne et la co-responsabilité.

Reste une question qu'on aborde trop rarement jusqu'ici : comment évaluer la gouvernance culturelle, sur la base de quels indicateurs?

Les travaux restent encore quelque peu balbutiants à ce sujet, malgré quelques bonnes initiatives de la part d'OCPA (Observatoire sur les Politiques Culturelles en Afrique) ${ }^{25}$ et d'Interarts.

Le programme CultureWatchEurope, quant à lui, vise à concevoir la culture comme « l'âme de la démocratie », en apportant un témoignage novateur sur les évolutions en matière de culture, de patrimoine et de médias en Europe. Il fournit une valeur ajoutée aux outils d'information, programmes et méthodes mis en place par l'Organisation en les reliant les uns aux autres et en invitant la société civile à y contribuer. Il est fondé sur des valeurs, réactif et conscient des enjeux : il a pour mission de mettre en relief les évolutions, les difficultés rencontrées et les bonnes pratiques, de manière à améliorer la gouvernance culturelle et la définition des politiques en la matière dans toute l'Europe, dans le respect des droits de l'homme.

Signalons aussi tout le travail qui se fait autour de l'Agenda 21 de la Culture, pour qui la gouvernance culturelle est l'un des cinq thèmes fondamentaux de sa réflexion et de son action. Si tout le monde semble tom-

24. Ces rôles s'ajoutent évidemment aux rôles plus « traditionnels » des pouvoirs publics : protection et mise en valeur des patrimoines, assurer les infrastructures et équipements culturels de base, veiller au financement de l'aide à la création artistique et des projets culturels, développer le cadre juridique et réglementaire.

25. Cfr notamment le projet de recherche sur les indicateurs culturels du développement humain, par Paul Nchoyi Nkiwi, OCPA, 2006. 
ber d'accord aujourd'hui sur la nécessité d'un monitoring indépendant, par exemple par des Observatoires reconnus, faudrait-il aller plus loin et prévoir, entre autres, des Agences de Notation de la gouvernance culturelle et une Agence Ranking pour les oNG qui y participent?

Inutile d'aller aussi loin pour le moment, mais ce qu'il faudra, en tout cas, c'est intégrer cette gouvernance culturelle dans les réformes administratives et la mettre désormais au centre des relations entre la politique et l'administration, entre le centre et les périphéries et prévoir les dévolutions et délégations de pouvoir nécessaires.

\section{Pour ne pas conclure...}

Aujourd'hui, un peu partout dans le monde, la gouvernance culturelle existe. Elle unit en quelque sorte le moment utopique au moment pragmatique. Certes, elle est « machine d'optimisation » technocratique et « boîte à outils » pour l'ingénierie et le management culturels. Mais elle comporte aussi une vision de la société et elle démontre que la culture est un lieu de déploiement des intelligences, des créativités et des créations de sens et qu'elle peut devenir une matrice essentielle de notre rapport au présent et à l'avenir. Il est donc important qu'au-delà du consensus technocratique et post-idéologique qu'il semble y avoir aujourd'hui concernant la gouvernance culturelle, nous puissions pleinement en développer la force politique, de démocratisation et de citoyenneté.

Nous disposons aussi de textes qui donnent un sous-bassement « idéologique » et juridique international :

- La Déclaration Universelle sur la diversité culturelle (2001) et la Convention sur la protection et la promotion de la diversité des expressions culturelles (2005) : il est sans doute important de souligner que ces deux textes de l'UNESCO prévoient une implication/responsabilisation importante de la société civile. ${ }^{26}$

- L'Agenda européen de la Culture (2007), de l'Union Européenne.

26. Cfr les « coalitions » pour la diversité culturelle qui se sont formées dans nombre de pays. 
- L'Agenda 21 de la Culture (2004/2005), qui concerne prioritairement les pouvoirs locaux et régionaux.

Ce qui ressort néanmoins de ce qui précède :

- La gouvernance culturelle est désormais un thème de discussion central pour les politiques culturelles, mais aussi pour le développement humain en général. La recherche sur ce thème reste encore très lacunaire, tout comme les exemples de bonne pratique restent peu nombreux et souvent difficilement transposables de l'un à l'autre.

- Il ne saurait y avoir un « modèle » de gouvernance culturelle unique et il ne peut pas être imposé d'en haut.

- Avant d'être une « technique » de gestion du développement culturel, la gouvernance culturelle est une éthique, se fondant sur les droits culturels. Cette éthique joue tant dans les objectifs qu'elle se fixe que dans les processus qu'elle met en œuvre.

- Elle fait agir ensemble des modèles rationnels différents : économique et marché (compétition, prix, result oriented), social (cohésion et justice), politique (recherche de majorités et de consensus, dans une opposition droite-gauche), administration (réglementation, loyauté envers le pouvoir politique), arts et culture (créativité, besoins de financement, « temps » très différents de l'économique et du politique, process oriented).

- Les principes en sont importants : transparence, égalité des chances, notamment dans l'accès à la culture, participation, partenariat, coopération, co-responsabilité, coalitions et alliances, empowerment et renforcement des capacités, égalité des chances, prospective, promotion de la diversité culturelle et dialogue interculturel, droits culturels, etc.

- La gouvernance culturelle ne saurait se limiter au secteur culturel. Elle peut d'ores et déjà s'inspirer de ce qui se passe dans la coopération au développement ou dans le développement durable. Mais elle doit aussi intégrer dans sa propre démarche le développement et le développement durable. Dans ce contexte, il paraît important d'insister sur la cohérence des politiques. 


\section{Quelques références bibliographiques ${ }^{27}$}

Agenda 21 de la Culture (2004) : par la Cglu (Cités et Gouvernements Locaux Unis, Commission Culture), voir $<$ www.agenda21culture/ net>.

Bellina, S. ; H. Magra et V. De Villemeur (sous la direction de) (2008) : La gouvernance démocratique. Un nouveau paradigme pour le développement?, Karthala, Paris.

BENz, A. (Hrsg, 2006) : Governance im modernen Staat, Verlag für Sozialforschung, Wiesbaden.

Cahiers Françoise (2011) : La pensée politique, quels renouvellements?, La Documentation Française.

Castells, M. (1999) : L'Ere de l'Information (La société en réseaux; Fin du Millénaire ; le pouvoir de l'identité), Fayard, Paris.

Collegium International (2012) : Le monde n'a plus de temps à perdre. Appel pour une gouvernance mondiale solidaire et responsable, Les Liens qui Libèrent.

Colloque Cerisy (2000) : Prospective pour une gouvernance démocratique, coordonné par Heurgon, E et J. Landrieu, Aube, 2000.

CONSEIL DE L'Europe (2010) : Culture and Development Twenty Years after the Fall of Communism in Europe, Strasbourg.

CNuCED et UnCTad (2008) : Creative Economy Report 2008. The Challenge of Assessing the Creative Economy : towards informed Policy making.

Deutscher Bundestag (Hrsg, 2008) : Kultur in Deutschland. Schlussbericht der Enquête Kommission des Deutschen Bundestages, ConBrio.

Finlande, Ministère de l'Education (2006) : Fair Culture - Culture for Sustainable Development, Background Paper on Cultural Sector and Development Work in the Nordic Countries, Helsinki.

Fuinder, M. (2002) : «Governance in Whitehall», Public Administration n. 80.

Florida R. (2002) : The Rise of Creative Class, Basik Books, New York.

Folke Schuppert, G. (Hrsg, 2005) : GovernanceForschung. Vergewisserung über Stand und Entwicklungslinien, Nomos, Baden-Baden.

GarnhaM, N. (2005) : «From Cultural to Creative Industries. An Analysis of the Implications of the "Créative Industries" Approach to Arts and Media Policy Making in the UK». In : International Journal of Cultural Policy 11, 1.

27. Complétées en 2014 
Glaeser, E. (1998) : «Are Cities Dying?», Journal of Economic Perspectives, n. 12.

GrefFe, X. (2010) : «Quelle politique culturelle pour une société créative ?». In : Politiques et Pratiques de la Culture (sous la direction de Philippe Poirrier), La Documentation Française.

— et S. Pflieger (2009) : La politique culturelle en France, La Documentation Française, Paris.

Groupe de Fribourg (2007) : Déclaration sur les Droits Culturels, Fribourg et Genève, voir < www.unifr/ch/iiedh $>$, publications.

Hermet, G. ; A. Kazancigil et J.-F. Prud'homme (2005) : La gouvernance. Un concept et ses applications, Karthala.

Jacquet, P. ; J. Pisany-Ferry et L. Taubiana (2002) : Gouvernance mondiale, La Documentation Française.

Jouve, B. (2007) : Le "political rescaling» pour théoriser l'Etat et la compétition territoriale en Europe. In Faure, A. ; J-PH. Leresche ; P. Muller et S. Nahrath (dir.): Action publique et changement d'échelles: les nouvelles focales du politique, Paris, l'Harmattan, pp. 45-55.

Kazancigil, A. (2010) : La gouvernance, pour ou contre le politique, Armand Colin.

Koolman, J. (2003) : Governing as Governance, Sage, London.

LaAksonen, A. (2010) : Making Culture Accessible - Access, Participation in Cultural Life and Cultural Provision in the Context of Cultural Rights, Conseil de l'Europe, Strasbourg.

Lange, B. ; A. Kalandides ; B. Stöber et I. Wellmann (Hrsg, 2009) : Governance der Kreativwirtschaft, Transcript.

Latour, B. (2007) : Eine neue Soziologie für eine neue Gesellschaft, Frankfurt/Main.

Le Glatin M. (2007) : Internet, un séisme dans la culture, Editions de l'Attribut.

LÉvy, P. (1997) : L’intelligence collective, La Découverte, Paris

— (2002) : Cyberdémocratie, Odile Jacob, Paris.

March, J. et J. Olsen (1995) : Democratic Governance, The Free Press, New York-London.

Martinell, A. (2010) : Gouvernance de la culture et diversité culturelle, Document de travail pour le Séminaire International sur Culture et Développement, Girona. 
MerkLe, K. (2010) : «Neue Initiativen des Europarates». In Kulturpolitische Mitteilungen, n. 129, Kulturpolitische Gesellschaft, Bonn.

Meyer-Bisch, P. et M. Bidault (2010) : Déclarer les droits culturels : commentaire de la Déclaration de Fribourg, Bruylant/Schulthess.

Moreau Defarges, Ph. (2003) : La gouvernance, puf, Paris.

O'Connell, H. et D. Souter (ed.) (1994): Good Governance. Report on a One.

PNud ; UndP (2004) : Rapport sur le développement humain : la liberté culturelle dans un monde diversifié.

Rosenau, J. N. et E. O. Czempiel (dir.) (1992) : Governance without Government : Order and Change in World Politics, Cambridge University Press, Cambridge.

SAEz, G. (2004) : « Gouvernance culturelle territoriale ». In : Institutions et vie culturelles, La Documentation Française.

SCheytт, O. (2008) : Kulturstaat Deutschland. Plädoyer für eine aktivierende Politik, Transcript.

Touraine, A. (2005) : Un nouveau paradigme. Pour comprendre le monde d'aujourd'hui, Fayard.

Tremblay, G. (2008) : « Industries culturelles, économie créative et société de l'information ». In Global Media Journal, Canadian Edition, volume 1 , Issue 1.

Unesco (2001) : Déclaration universelle sur la diversité culturelle, Paris.

- (2005) : Vers les sociétés du savoir, Paris.

-(2005) : Convention sur la protection et la promotion de la diversité des expressions culturelles, Paris.

- (2009) : Investir dans la diversité culturelle et le dialogue interculturel, Paris.

Union Africaine (uA, 2006) : Charte de la Renaissance Culturelle Africaine, Khartoum.

Union Européenne (ue, 2007) : Agenda européen de la Culture à l'ère de la mondialisation, Bruxelles.

Wagner, B. (2009): Governance in der Kulturpolitik. Vortrag im Center for Advanced Studies der Theaterwissenschaften München.

ZIAI, A. (2006): Zwischen Global Governance und Post-Development, Westfälisches Dampfboot. 